\title{
Free association within categories as a function of typicality
}

\author{
BERT ZIPPEL \\ City University of New York, Hunter College, New York, New York 10021
}

\begin{abstract}
The experiment attempted to determine whether a common retrieval cue operates to guide free associations when the stimulus material consists of pairs of category associates varying in typicality. Individuals were presented 48 pairs of words that were the $3 \mathrm{rd}$ and $4 \mathrm{th}, 9 \mathrm{th}$ and $10 \mathrm{th}$, or 17th and 18th associates of category labels, under free association instructions or instructions that stressed that each pair came from a common category. The different instructions produced no performance differences. The high- and medium-typicality stimulus pairs elicited the category label or some other highly typical member of the category. The lower typicality stimuli also produced category label responses or elicited lower typicality responses. Level of stimulus typicality did not affect total relevant responses or amount of category label responses.
\end{abstract}

Slamenka (1972) found that cuing recall of items of categorized lists increased access to categories but not recall of items within categories. Roediger (1973) and Rundus (1973) found that the more instances presented as cues, the less likely that remaining items would be recalled. Rundus (1973) argues that such evidence indicates that items within a categorized list are not associated with each other but with a common retrieval cue. The more cues presented, the greater the strength of association to the retrieval cue, and the less likely that other individual elements will be retrieved. Thus, verbal material is organized hierarchically, and the presentation of categorized items will invoke a control retrieval cue rather than further instances of items within the category. Postman (1975) warns, however, that this schema may be limited to categorized lists.

Restle (1974) also argues that the organization of verbal material in memory is hierarchical. However, he supposes that retrieval will proceed through lower levels of response production before transferring control of the flow to higher level cues.

The impetus of the present study was an attempt to determine whether mediation via a common retrieval cue operates in a free association context. Battig and Montague (1969) have reported normative responses to various category labels in which they noted that the frequency of responses given within a category correlate with the rank order of the responses to the category label. That is, the more frequently given response tended also to be the first response given by their respondents. Thus, if Restle's (1974) conception holds for free association production, students could be expected to give less frequent responses within a category than those with which they are primed. If respond-

Requests for reprints should be sent to Bert Zippel, Box 389 , Department of Psychology, Hunter College of the City University of New York, 695 Park Avenue, New York, New York 10021. ing is through a common retrieval cue, then, regardless of the response strength of the primary stimuli, an individual should tend to respond with high-frequency associates of the common retrieval cue.

\section{METHOD}

\section{Subjects}

The participants in the experiment were 32 undergraduate students who performed as part of a course requirement. All data were collected within one class session.

\section{Procedure}

For each of the 56 categories for which Battig and Montague (1969) reported normative responses, except 8 randomly chosen categories that were reserved for another study, the 3 rd and 4 th responses, the 9 th and 10 th responses, and the 17 th and 18th responses were selected to represent high-, medium-, and low-typicality pairs, respectively. Three forms were constructed with the high-, medium-, or low-typicality pairs for each category randomly assigned and ordered to each form with the restriction that each resulting form consist of an equal number of high-, medium-, and low-typicality pairs. The pairs were typed in capital letters, double spaced, with approximately a 1-in. blank line to the right of each pair, and numbered from 1 to 48 . The first 25 pairs appeared on one page, with the remaining pairs on a second page. In addition, the order or presentation of each pair of words was randomized for each form. For each of the three forms, one of two sets of instructions was printed on the first page above the first pair of words. One-half of the participants were instructed to write the first other word that came to mind for each pair of words. The others were instructed that each pair of words came from a common category and that the task was to write the first other word that came to mind from the same category. The former instruction is referred to as the free response condition, and the latter instruction is referred to as the category response condition.

\section{RESULTS}

The responses of the students were coded as the rank order recorded in the Battig and Montague (1969) norms. In cases in which the category label was given, the response was coded as a rank of zero. For each 
Table 1

Percent Germaine, Proportion Category Labels, and Median and Mean Rank of Within-Category Responses as a Function of Stimulus Pair Typicality

\begin{tabular}{lccc}
\hline & \multicolumn{3}{c}{ Typicality of Stimulus Pairs } \\
\cline { 2 - 4 } Measures of Germaine & $\begin{array}{c}\text { Associates } \\
\text { Responses }\end{array}$ & $\begin{array}{c}\text { Associates } \\
9 \& 4\end{array}$ & $\begin{array}{r}\text { Associates } \\
17 \& 10\end{array}$ \\
\hline Percent Germaine & 67.5 & 66.2 & 61.5 \\
Proportion Label & .33 & .34 & .29 \\
Median Rank & 4.1 & 3.4 & 10.0 \\
Mean Rank & 12.8 & 12.6 & 25.2 \\
\hline
\end{tabular}

student, the mean and the median ranks were determined for the high, medium, and low stimulus word pairs. Only category label or normative category member responses were included as germaine. The proportion of germaine responses that were category labels and the total number of extranormative and omitted responses were also determined for the three levels of stimulus pairs for each student. For each of the above-mentioned statistics, a two-factor analysis of variance with the two levels of the instruction factor and three levels with repeated measures of the stimulus pair factor was performed. There were no significant differences among the conditions as a function of the number of germaine responses. The analysis of variance for the proportion of germaine responses that were category labels also produced no statistically significant differences, although there was a consistent trend across all three levels of stimulus pairs for the category instruction to produce fewer category labels as responses.

The analyses of variance for both the mean and median rank of category responses produced patterns of significance similar to each other. In neither case did the difference in instructions or the interaction of instructions and differences in stimulus pairs result in significant differences. However, for the measure of median $[F(2,60)=3.32, p<.05]$ and mean rank category responses $[F(2,60)=9.99, p<.01]$, the factor of differences in stimulus pairs was significant. Table 1 presents the mean and median data for these three conditions, along with the data of the other analyses mentioned above. The data indicate that when the 17 th and 18 th associates of a category label are presented as a stimulus pair, the students respond with the label of the category or with a less typical member of the category than if they were presented with a more typical pair of category associates.

\section{DISCUSSION}

These results support the contention that highly typical instances of a category elicit the label of the category or some other highly typical member of the category. This is consistent with the conception of a prototypical category response or typicality as developed by Rips, Shoben, and Smith (1973) and Rosch $(1974,1975)$ using other paradigms. However, with less typical category members as stimuli, these data indicate that if the category label is not chosen, another less typical member of the category will be produced. There is little or no difference in correct comprehension of the relevant category or in reliance on the name of the category as a response; but, less typical stimulus pairs elect less typical, but equally germaine, category members than do highly typical pairs of stimuli.

The proportion of category-relevant responses or category label responses would probably decrease if even less typical pairs of words were used as stimuli. However, even greater proportions of within-category responses might be elicited if triplets rather than pairs of words were used as stimuli. The latter technique might also produce less between-subjects variability, which would facilitate a more sensitive measure of the effect of differing instructions than was obtained with the present design.

The data, however, do permit the rejection of the notion that pairs of category instances will elicit less typical instances as responses. Also rejected is the notion that responses will be directly mediated by the category label regardless of the typicality of the category instances chosen as stimuli. The data do indicate that some students rely on category labels as responses more than others do. There is a skewed distribution of reliance on category labels, with one-third of the students reporting less than $20 \%$ reliance on such responses, one-third between $20 \%$ and $40 \%$, and one-third above $40 \%$. No trend within these three groupings is evident with regard to either independent variable. The data were not analyzed to determine whether category labels as responses were more likely to occur with some pairs of words as stimuli than other pairs. As indicated above, sampling over a broader range of typicality is necessary to determine the relation between proportion of category labels as responses and typicality of other germaine category responses.

\section{REFERENCES}

Battig, W. F., \& Montague, W. E. Category norms for verbal items in 56 categories: A replication and extension of the Connecticut category norms. Journal of Experimental Psychology Monograph, 1969, 80, 1-46.

Postman, L. Verbal learning and memory. Annual Review of Psychology, 1975, 26, 291-335.

Restle, F. Critique of pure memory. In R. L. Solso (Ed.), Theories in cognitive psychology: The Loyola symposium. New York: Wiley, 1974.

Rips, L. J., Shoben, E. J., \& Smith, E. E. Semantic distance and the verification of semantic relations. Journal of Verbal Learning and Verbal Behavior, 1973, 12, 1-20.

Roediger, H. L. Inhibition in recall from cuing with recall targets. Journal of Verbal Learning and Verbal Behavior, 1973, 12, 644-657.

Rosch, E. H. Universals and cultural specifics in human categorization. In R. Brislin, W. Lonner, \& S. Bochner (Eds.), Crosscultural perspectives on learning. London: Sage Press, 1974.

Rosch, E. H. Cognitive reference points. Cognitive Psychology, $1975,1,532.547$.

Rundus, D. Negative effects of using list items as recall cues. Journal of Verbal Learning and Verbal Behavior, 1973, 12, 43-50.

Slamenka, N. J. The question of associative growth in the learning of categorized materials. Journal of Verbal Learning and Verbal Behavior, 1972, 11, 324-332.

(Received for publication September 21, 1979.) 